\title{
SUMMARY BOX
}

\section{What was known before?}

- Worldwide childhood blindness is a major cause of needless lifelong morbidity particularly in low and middle income countries (LMICS)

- The Red reflex test is a highly sensitive yet simple means to detect congenital cataract the main cause of treatable childhood blindness in LMICs

- The Red reflex is assessed using a traditional direct ophthalmoscope (TDO)

- TDOs are expensive to purchase and maintain and are consequently rarely used by health care workers in LMICs

\section{What this study adds?}

- The diagnostic performance of a new low cost solar powered direct ophthalmoscope (Arclight) is equivalent to that of a more expensive TDO in detecting abnormal red reflexes in simulated eyes

- The majority of Malawian health care workers preferred the Arclight over the TDO

- The additional features of such a frugal, consumable independent, solar powered and portable device are especially pertinent in Malawi one of the poorest countries in the world 


\section{TITLE:}

\section{Comparative evaluation of a low cost ophthalmoscope (Arclight) for red reflex assessment amongst health care workers in Malawi}

Dooley $\mathrm{E}^{1}$, O Kousha ${ }^{2}$, Msosa J ${ }^{3}$, Ndaule $\mathrm{E}^{3}$, Abraham $\mathrm{C}^{4}$, Kayange $\mathrm{P}^{5}$, Parr J ${ }^{6}, \mathrm{O}^{\prime} \mathrm{Hare}^{7}$, Blaikie $A^{8}$,

1. Medical student, University of Leeds, Leeds, United Kingdom

2. Ophthalmology Trainee, Department of Ophthalmology, NHS Fife

3. Department of Ophthalmology, Kamuzu Central Hospital, Lilongwe

4. Lead Optometrist, Department of Optometry, College of Allied Health Sciences, Lilongwe

5. Lions First Sight Eye Unit, Queen Elizabeth Central Hospital, Blantyre

6. Programme Lead iBSc International Health, University of Leeds, United Kingdom

7. School of Public Health, University of Malawi

8. School of Medicine, University of St Andrews, United Kingdom

\section{Corresponding Author - Obaid Kousha okousha@gmail.com}




\section{ABSTRACT}

We compared the diagnostic performance and ease of use of a new solar powered low-cost Arclight ophthalmoscope (AO) to a more expensive traditional direct ophthalmoscope (TDO) (Keeler Professional V.2.8) in detecting abnormal red reflexes in simulated eyes.

Both devices were used by 19 optometry students and 17 paediatric doctors based in the Kamuzu Central Hospital Campus in Lilongwe, Malawi. Participants examined 4 normal and 4 abnormal red reflexes using the two devices in random order. We scored the participants on their ability to identify clinical signs and make a diagnosis. Participants scored each device for "ease of use". There was no statistically significant difference in diagnostic performance or "ease of use" between the $A O$ and the TDO when attempting to detect abnormal red reflexes in simulated eyes.

We conclude that $A O$ is an inexpensive yet equally effective alternative to the TDO in detecting red reflexes and due to its low cost, portability and consumable independence is well suited for use in low and middle income countries. 


\section{INTRODUCTION}

Reduction of childhood blindness is a World Health Organisation priority (1). Although the prevalence of childhood blindness is estimated to be ten times lower than in adults, the number of disability-adjusted life years is greater with up to one-half of childhood blindness being preventable or treatable (1). Early diagnosis offers the best opportunity for effective prevention and treatment $(2,3)$.

The red reflex (RR) test is a simple yet highly sensitive means to detect the majority of visually impairing conditions of childhood including corneal scaring, cataract and retinoblastoma but also refractive error, anisometropia and strabismus (4-6). In regions where systematic screening for childhood eye disease is limited the RR test can be used opportunistically by a range of primary and mid-level health care workers to detect otherwise cryptic disease for early treatment and better outcomes $(7,8)$.

The RR test can be easily performed with minimal training using a direct ophthalmoscope. The traditional direct ophthalmoscope (TDO) is however rarely available outside of specialist eye units in low and middle-income countries (LMICS). They are typically expensive and difficult to maintain in working order as they depend upon a supply of costly and hard to find bulbs and batteries (9). These limitations make the TDO largely inaccessible to most primary and mid-level healthcare workers in LMICs where the majority of childhood blindness is found. To address the limitations of the TDO the 'Arclight' direct ophthalmoscope (AO) (9) has been developed.

The AO is a low cost, consumable independent solar-powered ophthalmoscope that has been developed specifically to meet the needs of health care workers in LMICs (9). The AO utilises a light emitting diode (LED) with an integrated photovoltaic solar panel charging a slim internal rechargeable battery. Simplification of the design by placing the LED on the front of the device below the sight hole has allowed miniaturisation (110 mm long $\times 26 \mathrm{~mm}$ wide $\times 9 \mathrm{~mm}$ thick, $18 \mathrm{~g}$ ) making it 
easily portable (Figure 1). In addition to functioning as a direct ophthalmoscope the AO has an integrated magnifying loupe which can also function as an otoscope. Importantly when sold in bulk it can be purchased for $£ 10$ per unit. Initial evaluation studies have shown the device to be at least as effective as a traditional device and perceived as easier to use (10 -13).

The AO consequently offers the opportunity to expand direct ophthalmoscopy access in low resource settings and increase RR assessments amongst young children reducing the burden of needless blindness and death. No study has however yet evaluated the specificity and sensitivity of the AO when in the RR test compared to a TDO. This study therefore aims to compare the AO with a TDO in assessing the findings of the RR test on simulated eyes when used by health care workers in Malawi.

\section{METHOD}

The study was conducted at the Kamuzu Central Hospital Campus in Lilongwe, Malawi. 19 optometry students from the School of Optometry and 17 paediatric doctors from the Paediatric Department of were recruited.

A short presentation was given outlining the study including the findings of normal and abnormal RR tests on the simulation eyes. Participants then examined eight simulation eyes containing four abnormal and four normal red reflexes. There were 4 possible red reflex abnormalities designed to mimic specific pathologies (Figure 2): White reflex (retinoblastoma), central dark opacity (polar cataract), peripheral wedge shaped opacity (cortical cataract) and a misshapen pupil (coloboma). The order of the eight simulated eyes examined and the initial device used were chosen using a random number generator. Once every simulated eye in the first run was examined and findings documented the same simulated eyes were rearranged into a different random order. The participant then examined a second array of 8 simulated eyes with the other device. After examining 
the simulation eyes the participants recorded an ease of use score for both devices using a Likert Scale with 1 being "very easy to use" and 5 being "very hard to use".

\section{Data analysis}

Data were entered into a spread sheet and analysed using IBM SPSS Statistics for Windows, Version 22. All tests were two tailed with type I error set at $\alpha=0.05$. Paired t-test was used to analyse the paired parametric data from each participant. Ordinal data from Likert Scale scores for the two devices were compared using Wilcoxon Signed-Rank test. Specificity, sensitivity, positive and negative predictive values were calculated for each device and the paired outcomes were compared using McNemar's test.

\section{Ethical Approval}

Ethical approval was given from the University of Leeds, University of St Andrews and the College of Medicine Research and Ethics Committee, Malawi.

\section{RESULTS}

\section{Diagnostic performance}

The ability to identify abnormal RR tests was comparable between the two devices (Table 1 ) with the mean score for correct diagnosis 6.78 out of 8 for the TDO and 6.53 out of 8 for the AO. The difference was not statistically significant $(p=0.203)$. The breakdown of the results showed that the optometry students demonstrated a trend to better diagnostic skills with both devices compared to the paediatric doctors but these differences were not statistically significant (Table 1). 
Table 1: Healthcare professional's scores using the two devices

\begin{tabular}{|c|c|c|c|}
\hline & \multicolumn{2}{|c|}{$\begin{array}{l}\text { Mean diagnostic score out of a } \\
\text { mximum of } 8\end{array}$} & \multirow[t]{2}{*}{$p$-value } \\
\hline & $\mathrm{AO}$ & TDO & \\
\hline Doctors \& Optometrists & 6.53 & 6.78 & $p=0.203$ \\
\hline Device & Doctors & Optometrists & \\
\hline $\mathrm{AO}$ & 6.35 & 6.68 & $p=0.587$ \\
\hline TDO & 6.52 & 7 & $p=0.460$ \\
\hline $\mathrm{AO}+\mathrm{TDO}$ & 6.44 & 6.84 & $p=0.355$ \\
\hline
\end{tabular}

As expected the differences in sensitivity, specificity, positive and negative predictive values of $A O$ and TDO in detecting pathology in simulated eyes were also not statistically significant (Table 2). However, the AO had a trend to be slightly better on sensitivity and the TDO on specificity. Importantly, the negative predictive values of both devices were well above $80 \%$.

Table 2: Diagnostic performance of the two devices

\begin{tabular}{lll}
\hline & AO $(95 \% \mathrm{Cl})$ & TDO $(95 \% \mathrm{Cl})$ \\
\hline Sensitivity & $85.4 \%(79.7 \%-91.2 \%)$ & $83.5 \%(77.2 \%-89.4 \%)$ \\
Specificity & $77.8 \%(71.0 \%-84.6 \%)$ & $86.1 \%(80.4 \%-91.8 \%)$ \\
Positive predictive value & $79.4 \%(73.0 \%-85.7 \%)$ & $85.7 \%(79.0 \%-91.5 \%)$ \\
Negative predictive value & $84.2 \%(78.0 \%-90.4 \%)$ & $83.7 \%(77.8 \%-89.7 \%)$ \\
\hline
\end{tabular}

McNemar's Test showed the difference in the proportions listed above between the two devices were statistically insignificant, $p$-value $=0.211$

No learned effect was observed as there was no statistically significant difference between the average correct score for the first and second rounds (6.66 vs 6.63 respectively $p$-value $=0.95$ ).

\section{Ease of Use}

Overall there was a non-statistically significant $(p=0.64)$ trend for ease of use in favour of the AO with median Likert Scale score of 1 for the AO compared to 2 for the TDO. 20 participants (over half) ranked the $\mathrm{AO}$ as 'very easy to use' while only 11 (just under a third of participants) considered the 
TDO at this level. One participant ranked the $A O$ as 'very hard to use' while three participants ranked the TDO at this level.

\section{DISCUSSION}

Most direct ophthalmoscopes are designed for users working in developed regions of the world; they are typically expensive, dependent on often hard to find consumables and are increasingly unnecessarily complex. As a consequence, TDOs are rarely available in low income health care systems and even if they are present they are often in a non-functional state like many other medical devices found in hospitals of resource poor regions (14). The AO has been specifically designed to address the barriers to access of TDOs in LMICs. This study has shown that it has an equivalent diagnostic performance in identifying abnormal red reflex appearances in simulated eyes compared to a TDO amongst Malawian healthcare workers. Despite its low cost and simple design, the AO was preferred by the majority of study participants and was considered just as easy to use.

We have shown that the AO is an effective diagnostic tool amongst health care workers in Malawi where the additional features of being low-cost, consumable independent, extremely portable, compact and rugged are especially pertinent. These features make the $A O$ an ideal frugal yet effective device for LMICs as recommended by both the Vision 2020 initiative and the Lancet Commission on Technologies for global health $(15,16)$. The findings of this study are supported by others. Blundell and colleagues demonstrated that the AO is as effective as the TDO in diagnosing diabetic retinopathy in a simulated eye study (11) amongst health care workers also in Malawi, and Moin et al replicated these results on human subjects in Pakistan (12). In Tanzania, Lowe et al showed that the AO performs as well as the TDO in assessing the appearance of the optic nerve in human subjects (10). Additionally, in both these two studies the participants rated the $A O$ as easier of use. This may reflect the simplified, intuitive design and avoidance of 'feature creep' which afflicts and limits the usability of many medical devices (16). 
Furthermore, unlike telemedicine approaches that use more expensive dedicated retinal or mobile phone camera devices (17), the AO empowers healthcare workers to make independent on the spot diagnoses strengthening the human resource and thus further satisfying the three pillars of the Vision 2020 initiative (18).

The main limitation of this study is that a simulation eye does not adequately replicate the examination of a young and potentially uncooperative child. Therefore now that this initial study has been performed further studies should evaluate the device in assessing the RR in real patients and in particular babies.

In view of the growing positive evidence base (9-13) the International Association of Prevention of Blindness (IAPB) has endorsed the Arclight by including it on the 'Standard List' of recommended device for use in LMICs with over 10,000 devices currently in use around the world (19). Our study adds further evidence that adoption of the Arclight by health care systems in LMICs can assist in the diagnosis of eye disease reducing needless blindness in these regions where the burden is greatest yet access to diagnostic devices least.

\section{ACKNOWLEDGEMENT}

We would like to acknowledge the assistance of the staff of the School of Optometry, College of Allied Health Sciences and the department of Paediatrics at the Kamuzu Central Hospital Campus in Lilongwe, Malawi and in particular Dr Ajib Phiri the Assistant Dean for his continued support in this work.

\section{COMPETING INTERESTS}

We have read and understood the BMJ policy on competing interests and declare the following interests: $A B$ is seconded to the University of St Andrews from NHS Fife. The University owns a social enterprise subsidiary company, for which $A B$ acts as an unpaid adviser. The social enterprise business sells the Arclight to users in high resource countries with all profits being used to fund 
distribution and education exercises of the device in low income countries via the Global Health Implementation team at the University of St Andrews.

\section{REFERRENCES:}

1. WHO. Global Initiative for the Elimination of Avoidable Blindness, Action Plan 2006-2011. [Online] 2007. [Date accessed 13/02/2019] Available from: http://www.who.int/blindness/Vision2020 report.pdf

2. Gilbert C, Foster A. Childhood blindness in the context of VISION 2020 - The Right to Sight. Bulletin of the World Health Organization 2001; 79: 227-232.

3. Wan MJ, VanderVeen DK. Eye disorders in newborn infants (excluding retinopathy of prematurity). Arch Dis Child Fetal Neonatal Ed 2015; 100: F264-9.

4. American Academy of Pediatrics. Red reflex examination in neonates, infants, and children. Pediatrics 2008; 122: 1401-1404.

5. Sun M, Me A, Li F, Cheng K, Zhang M, Yang H et al. Sensitivity and Specificity of Red Reflex Test in Newborn Eye Screening. J Pediatr 2016; 179: 192-196.

6. Paysee EA, Williams GC, Coats DK, Williams EA. Detection of red reflex asymmetry by pediatric residents using the Brukner reflex Versus the MTI photoscreener. Pediatrics 2001; 108: e74; doi:10.1542/peds.108.4.e74

7. Foster A, Yorston D. Corneal ulceration in Tanzanian children: relationship between measles and vitamin A deficiency. Trans $R$ Soc Trop Med Hyg 1992; 86: 454-455.

8. Tuli SY, Giordano BP, Kelly M, Fillipps D, Tuli SS. Newborn with an absent red reflex. J Pediatr Health Care 2013; 27: 51-55. doi:10.1016/j.pedhc.2011.10.010

9. Blaikie A, Sandford-Smith J, Tuteja SY, O'Callaghan C. Arclight: a pocket ophthalmoscope for the 21st century. BMJ 2016; 355:i6637. doi:https://doi.org/10.1136/bmj.i6637 
10. Lowe J, Cleland CR, Mgaya E, Furahini G, Gilbert CE, Burton MJ et al. The arclight ophthalmoscope: a reliable low-cost alternative to the standard direct ophthalmoscope. J Ophthalmol 2015; e-pub 17 Sep 2015; doi:10.1155/2015/743263

11. Blundell R, Roberts D, Fioratou E, Abraham C, Msosa J, Chirambo T et al. Comparative evaluation of a novel solar powered low-cost ophthalmoscope (Arclight) by eye healthcare workers in Malawi. BMJ Innov 2018; 4: 98-102; doi:10.1136/bmjinnov-2017-000225

12. Moin M, Manzoor A, Riaz F. Arc light as an alternative approach to diagnose diabetic retinopathy (DR) at grass root level of health care system. Pak J Ophthalmol 2018; 34: 154162

13. Hey SY, Buckley JC, Shahsavari S, Kousha O, Haddow KA, Blaikie A et al. A mixed methods comparative evaluation of a low cost otoscope (Arclight) with a traditional device. Clin Otolaryngol 2019 (In press)

14. Perry L, Malkin R. Effectiveness of medical equipment donations to improve health systems: how much medical equipment is broken in the developing world? Med Biol Eng Comput 2011; 49: 719-22.

15. Howitt P, Darzi A, Yang GZ , Ashrafian H, Atun R, Barlow J et al. Technologies for global health. Lancet 2012; 380: 507-35; doi:10.1016/S0140-6736(12)61127-1

16. DePasse JW, Caldwell A, Santorino D, Bailey E, Gudapakkam S, Bangsberg D et al. Affordable medical technologies bring Value-Based Design into global health. BMJ Innov 2016;2:4-7

17. Chee R, Darwish D, Fernanadez-Vega A, Patel S, Jonas K, Ostmo S et al. Retinal Telemedicine. Curr Ophthalmol Rep 2018; 6: 36-45

18. Resnikoff S, Kocur I, Etya'ale DE , Ukety TO. Vision 2020 - the right to sight. Ann Trop Med Parasitol 2008; 102(Suppl 1): 3-5; doi:10.1179/136485908X337409

19. International Association of Prevention of Blindness (IAPB). Standard list. [Date accessed 19/05/2019] Available from: https://iapb.standardlist.org/get-arclight/ 


\section{FIGURE LEGENDS}

Figure 1

Arclight ophthalmoscope: Design simplification include LED placed on the front of device just below the sight hole

Figure 2

Title: Normal and abnormal red reflexes in simulation eyes

Legend: a) Participant examining the simulation eye, b) normal red reflex, c) white reflex (retinoblastoma), d) central dark opacity (posterior polar cataract), e) a misshapen pupil (coloboma), and f) peripheral wedge shaped opacity (lamellar cataract). 